\title{
Electrochemotherapy as a new therapeutic strategy in advanced Merkel cell carcinoma of head and neck region
}

\author{
Daniele Scelsi ${ }^{1}$, Niccolò Mevioํㅜ, Giulia Bertino ${ }^{1}$, Antonio Occhini ${ }^{1}$, Valeria Brazzelli², \\ Patrizia Morbini ${ }^{3}$, Marco Benazzo ${ }^{1}$
}

\begin{abstract}
${ }^{1}$ Department of Otolaryngology Head Neck Surgery, ${ }^{2}$ Institute of Dermatology, ${ }^{3}$ Department of Molecular Medicine, Section of Pathology, University of Pavia, IRCCS Policlinico S. Matteo Foundation, Pavia, Italy
\end{abstract}

Radiol Oncol 2013; 47(4): 366-369.

Received 10 May 2013

Accepted 11 July 2013

Correspondence to: Daniele Scelsi, M.D., Department of Otolaryngology Head Neck Surgery, University of Pavia, IRCCS Policlinico S. Matteo Foundation, P.le Golgi 19, 27100-Pavia (PV), Italy. E-mail: daniele.scelsi@gmail.com

Disclosure: The authors have no conflict of interest to disclose.

The paper was presented at the $7^{\text {th }}$ Conference of Experimental and Translational Oncology, 20-24th April 2013, Portoroz, Slovenia (Www.ceto.si) co-organised and supported by COST TD1104 Action (www.electroporation.net).

Background. Merkel Cell Carcinoma (MCC) is a rare and aggressive tumour, arising from a cutaneous mechanoceptor cell located in the basal layer of epidermis, with poor prognosis. The treatment of choice for the initial stage of the disease is surgery and/or radiotherapy. The treatment of recurrent or advanced disease is still controversial.

Case report. We report a case of 84 years old woman with a recurrent $\mathrm{MCC}$ of the chin treated with electrochemotherapy (ECT). During the period of 20 months, four sessions of ECT were employed, which resulted in an objective response of the tumour and good quality of residual life.

Conclusions. Our case shows the effectiveness of ECT in the treatment of locally advanced MCC of the head and neck region in a patient not suitable for standard therapeutic options.

Key words: electrochemotherapy; head and neck cancer; Merkel cell carcinoma; palliative treatment

\section{Introduction}

Merkel Cell Carcinoma (MCC) is a rare and aggressive cancer described for the first time in 1972 by Toker et al. ${ }^{1}$ Clinically it is characterized by a rapidly increasing red or bluish nodule. It frequently occurs in the head and neck area $(41-50 \%)$, followed by upper and lower limbs (32-38\%) and trunk (12$14 \%)$. This neuroendocrine neoplasia arises from a cutaneous mechanoceptor cell (Merkel cell), located in the basal layer of epidermis. ${ }^{1}$ The annual incidence in the Caucasian population is 0.23 per 100,000 individuals and $61 \%$ of these cases are men. MCC affects more frequently elderly and immunecompromised patients with a previously history of damaging sun exposure. ${ }^{2}$ The prognosis is poor due to the fast local growth and high local recurrence, regional lymph node metastases and distant metastases rates, occurring even after the prompt treatment. ${ }^{3}$

The main treatment modality for the early stage tumours is surgery and/or radiotherapy, while chemotherapy with etoposide and cisplatin or carboplatin finds a role only in patients with systemic metastases. Due to the low incidence of MCC, the experiences with recurrent lesions or advanced stage tumours are scarce.,3

We report the case of a 84 years old woman affected by a extensive recurrence of MCC of the chin treated with multiple sessions of electrochemotherapy (ECT). 


\section{Case report}

A 84 years old woman with hypertension, chronic vascular disease, chronic cardiac ischemia was admitted because of a dome-shaped bluish skin lesion in her chin of $2 \times 2 \mathrm{~cm}$, characterized by rapid growth. A skin biopsy showed poorly differentiated cells with scarce cytoplasm and vesicular nuclei with inconspicuous nucleoli. High mitotic index and apoptotic figures were present. Immunohistochemical reactions were positive for cytokeratin 8 and 20 and neuroendocrine markers chromogranin, synaptophysin and CD56/N-CAM; TTF-1 was not expressed. A diagnosis of MCC was established (Figure 1).

Total body computed tomography scan and bone scintigraphy excluded mandibular infiltration, regional or systemic metastases. The lesion was staged cT2N0M0. The patient underwent surgical excision of the tumour, bilateral selective neck dissection (levels I-III) and reconstruction with radial forearm free flap. The histopathological examination of resected specimen confirmed a stage IIA (pT2N0M0) MCC and surgical margins free of tumour. The patient refused proposed postoperative radiotherapy, whereas the systemic therapy was not indicated due to co-morbidities.

Two months after the treatment, mass in the area of previous surgery extending into a submandibular region was documented. A biopsy of the mass confirmed recurrent MCC (Figure 2). The re-operation was not an option because of the tumour extension, and radiotherapy was again refused by the patient. She was offered a palliative treatment with ECT. The treatment was performed according to the standard operating procedures of the ESOPE. ${ }^{5}$ Intravenous bleomycin infusion of $15.000 \mathrm{IU} / \mathrm{m}^{2}$ was administered eight minutes before delivery of electric pulses under general anaesthesia by means of a hexagonal electrode (length $25 \mathrm{~mm}$ ) connected to a generator (Cliniporator ${ }^{\mathrm{TM}}$ - IGEA srl, Carpi, [MO], Italy). The lesion with $1 \mathrm{~cm}$ tumour-free margin was treated by multiple direct insertions of the electrode. The pulses were completed within 28 minutes after the infusion of bleomycin.

The patient was evaluated four weeks after the treatment: $50 \%$ reduction of the original tumour volume was observed, corresponding to a partial response according to RECIST criteria. ${ }^{6}$ The next ECT treatment resulted in $80 \%$ volume reduction compared to the original lesion (Figure 3).

Due to the good response and tolerability of the procedure, two additional ECT treatments were performed during the following sixteen months,

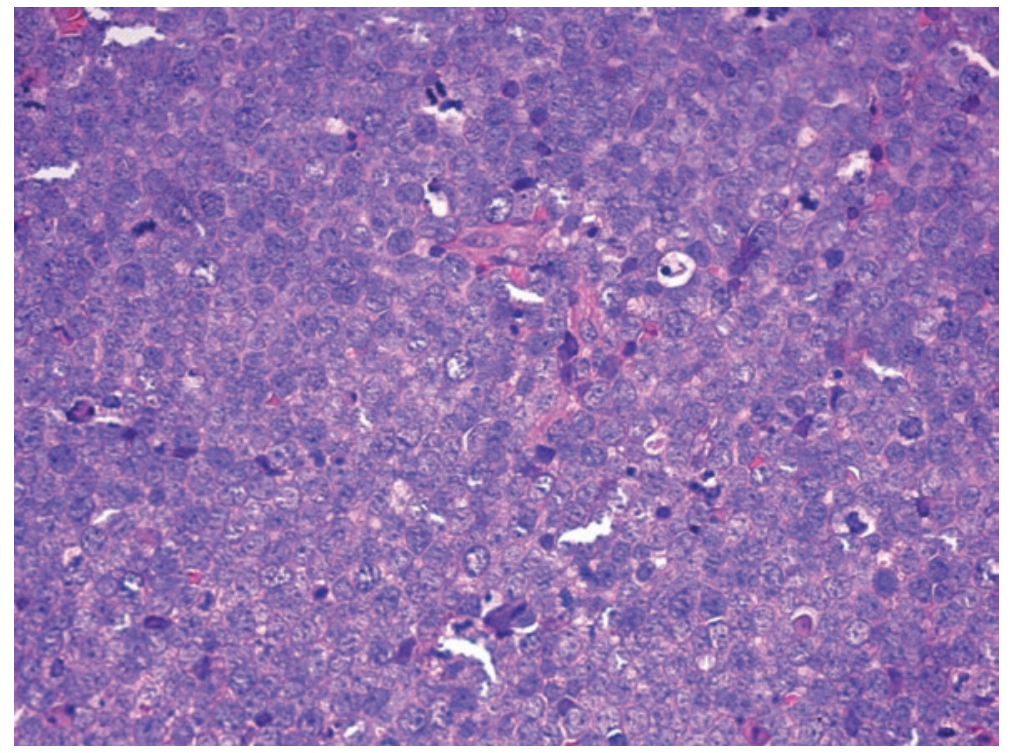

FIGURE 1. Tumour biopsy showing poorly differentiated cells with scarce cytoplasm and vesicular nuclei with inconspicuous nucleoli. High mitotic index and apoptotic figures were present. Immunohistochemical reactions were positive for cytokeratin 8 and 20 and neuroendocrine markers (chromogranin, synaptophysin and CD56/NCAM); TTF-1 was not expressed (H\&E stain, 40x).

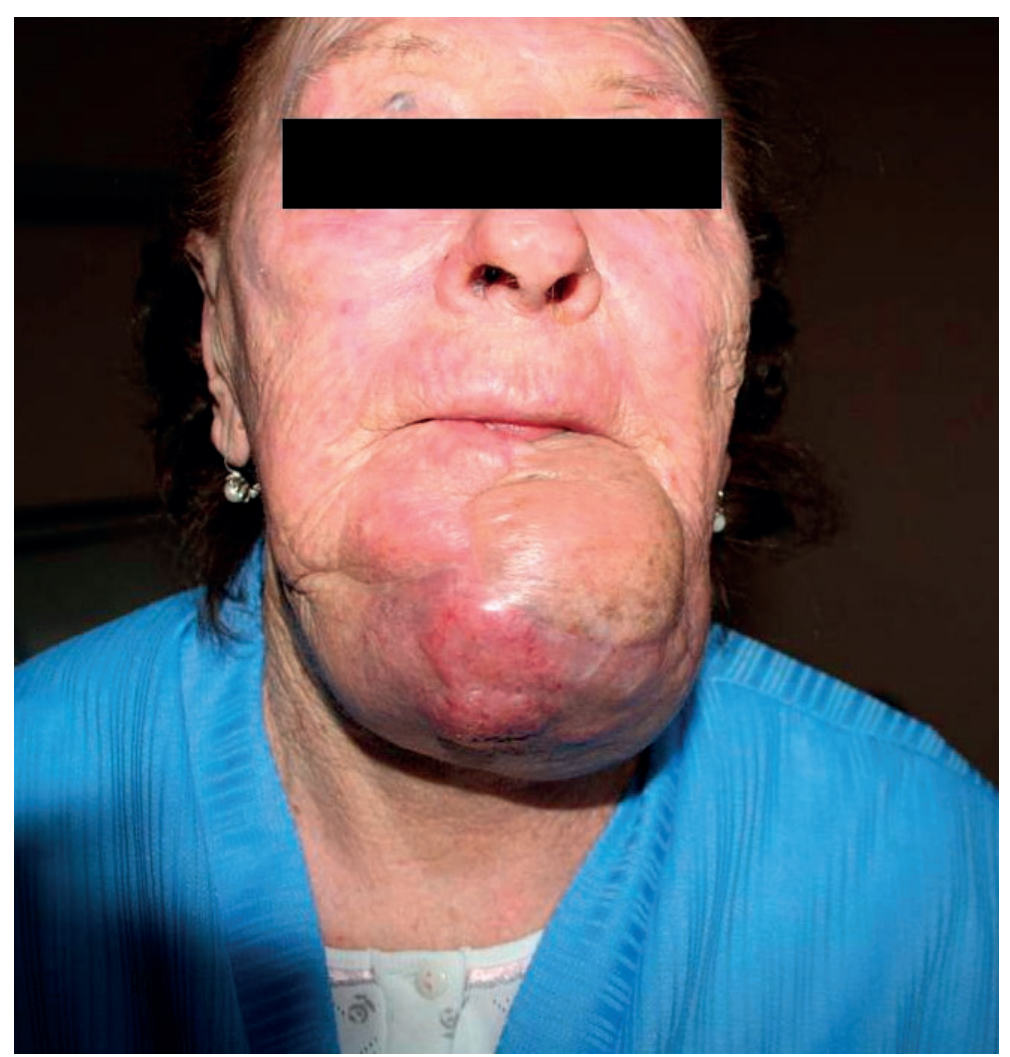

FIGURE 2. Before first electrochemotherapy: voluminous bluish lesion of the chin. 


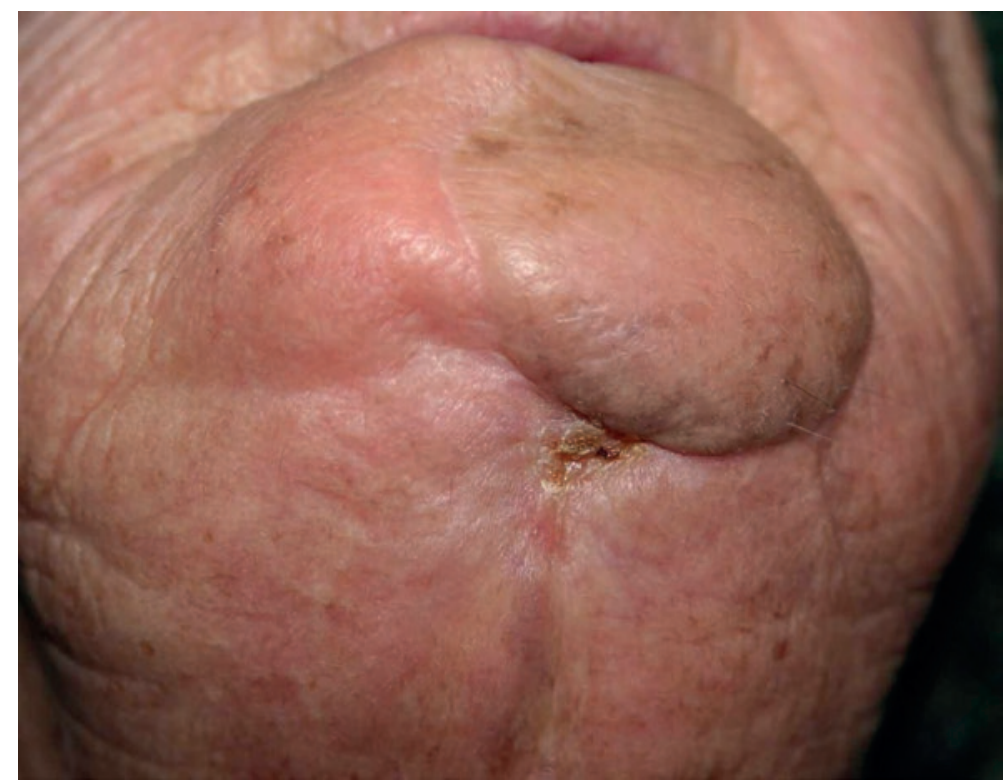

FIGURE 3. Reduction of $80 \%$ of the volume to the initial lesion after 2 treatments.

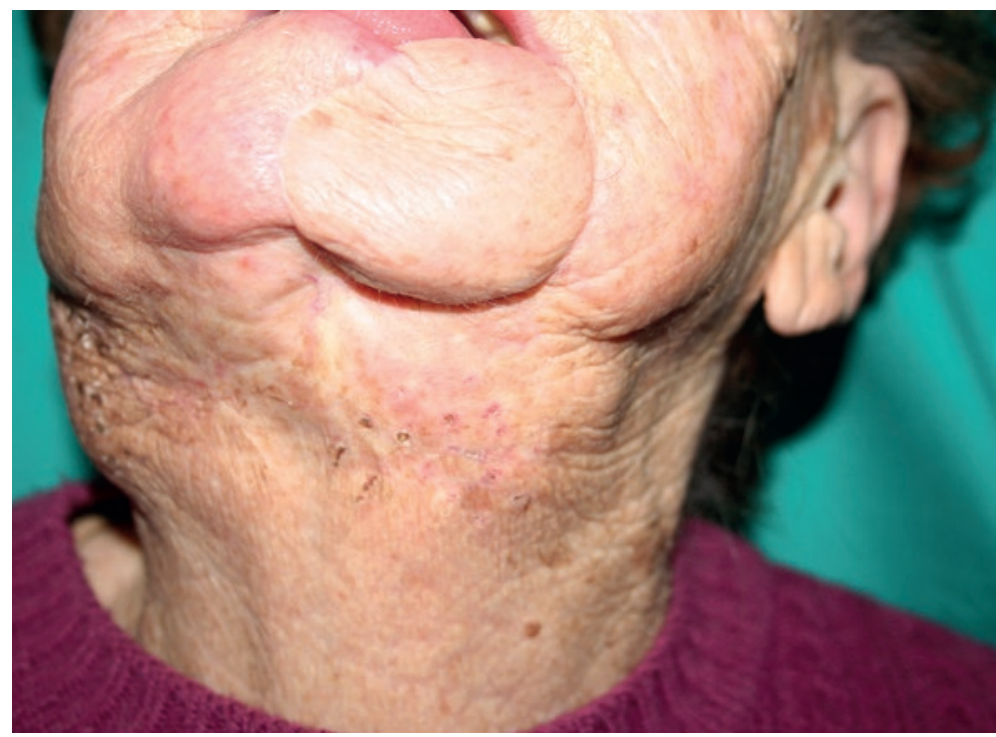

FIGURE 4. Follow up after four electrochemotherapy applications and 16 months.

which resulted in a good local control of the tumour (Figure 4).

Unfortunately, just after the fourth ECT treatment (and 20 months after the first ECT session), lung metastases were diagnosed. The patient died one month later due to heart failure which was unrelated to MCC.
No major complications were observed during or after ECT treatments. The only recorded side effect was moderate pain after the first ECT session, easily managed with peroral non-opioids. The patient reported a good quality of life until the last two months before she died when severe pain, controlled by opioids, appeared.

\section{Discussion}

The management of patients with MCC of the head and neck region is still challenging due to poor prognosis. According to the National Comprehensive Cancer Network guidelines ${ }^{7}$, surgery is commonly considered the treatment of choice and significantly improves the overall survival, if associated with adjuvant radiotherapy. ${ }^{8}$ In the head and neck area where it is difficult to obtain safety margins wide enough, radiotherapy can be the first treatment option. ${ }^{3}$ Because of the high incidence of occult regional metastasis, patients with clinical and radioghaphically negative necks should undergo elective dissection, irradiation, or preferably sentinel lymph node biopsy. Chemotherapy is an option for patients with an incurable recurrent, heavily pre-treated disease or for those with systemic metastases although without proven effect on the overall survival. ${ }^{9}$

In the last years, ECT has been proposed as a novel therapeutic weapon for the control of recurrent cutaneous, subcutaneous or mucosal neoplastic lesions of different histologies. ${ }^{5}$ In the literature, few data exist about the effectiveness of this procedure in the treatment of head and neck cancers; the reported rates of objective response (OR) seem promising, ranging from $56 \%$ to $100 \%$, depending on the tumour size. ${ }^{10-16}$ To the best of our knowledge there is only one case of MCC treated with ECT documented in the literature. The authors reported a complete response (CR) of the tumour to ECT after a follow up time of six months. ${ }^{12}$

The choice of using ECT in our patient was determined by the existing comorbidities and the patient's refusal of radiotherapy. With ECT we were able to reach a good local tumour control, with no significant adverse events, nor functional or esthetic, which resulted in a good quality of the rest of her life. This case demonstrates that ECT can be considered as an effective palliative treatment option for patients with recurrent or advancedstage tumour, not suitable for conventional treatments. ${ }^{10-14}$ 


\section{References}

1. Toker C. Trabecular carcinoma of the skin. Arch Dermatol 1972; 105: 107-10.

2. Pellitteri PK, Takes RP, Lewis JS Jr, Devaney KO, Harlor EJ, Strojan P, et al. Merkel cell carcinoma of the head and neck. Head Neck 2012; 34: 1346-54.

3. Pectasides D, Pectasides M, Economopoulos T. Merkel cell cancer of the skin. Ann Oncol 2006; 17: 1489-95.

4. Eng TY, Boersma MG, Fuller CD, Cavanaugh SX, Valenzuela F, Hermann TS. Treatment of merkel cell carcinoma. Am J Clin Oncol 2004; 27: 510-5.

5. Mir LM, Gehl G, Sersa G, Collins C, Garbay G-R, Billard V, et al. Standard operating procedures of the electrochemotherapy: Instructions for the use of bleomyicin or cisplatin administered either systemically or locally and electric pulses delivered by the Cliniporator ${ }^{\mathrm{TM}}$ by means of invasive or noninvasive electrodes. EJC Supp/ 2006; 4: 14-25.

6. Eisenhauer EA, Therasse P, Bogaerts J, Schwartz LH, Sargent D, Ford R, et al. New response evaluation criteria in solid tumours: revised RECIST guideline (version 1.1). Eur J Cancer 2009; 45: 228-47.

7. National Comprehensive Cancer Network, NCCN Guidelines TM, Version 1.2012, Merkel Cell Carcinoma. Available at: http://www.merkelcell.org/ usefullnfo/documents/NccnMcc2012.pdf

8. Tai PT, Yu E, Tonita J, Gilchrist J. Merkel cell carcinoma of the skin. J Cutan Med Surg 2000; 4: 186-95.

9. Suarez C, Rodrigo JP, Ferlito A, Devaney KO, Rinaldo A. Merkel cell carcinoma of the head and neck. Oral Oncol 2004; 40: 773-9.

10. Gargiulo M, Moio M, Monda G, Parascandolo S, Cubicotti G. Electrochemotherapy: actual considerations and clinical experience in head and neck cancers. Ann Surg 2010; 251: 773.

11. Panje WR, Hier MP, Garman GR, Harrel E, Goldman A, Bloch I. Electroporation therapy of head and neck cancer. Ann Otol Rhinol Laryngol 1998; 107: 77985

12. Curatolo $\mathrm{P}$, Mancini $\mathrm{M}$, Clerico $\mathrm{R}$, Ruggiero $\mathrm{A}$, Frascione $\mathrm{P}$, Di Marco $\mathrm{P}$, et al. Remission of extensive Merkel cell carcinoma after electrochemotherapy. Arch Dermatol 2009; 145: 494-5

13. Sersa G. The state-of-the-art of electrochemotherapy before the ESOPE study; advantages and clinical uses. EJC Supp/ 2006; 4: 52-9.

14. Mevio N, Bertino G, Occhini A, Scelsi D, Tagliabue M, Mura F, et al. Electrochemotherapy for the treatment of recurrent head and neck cancers: preliminary results. Tumori 2012; 98: 308-13.

15. Landström FJ, Nilsson CO, Crafoord S, Reinzenstein JA, Adamsson GB, Löfgren LA. Electroporation therapy of skin cancer in the head and neck area. Dermatol Surg 2010; 36: 1245-50.

16. Mali B, Miklavcic D, Campana LG, Cemazar M, Sersa G, Snoj M, et al. Tumor size and effectiveness of electrochemotherapy. Radiol Oncol 2013; 47: 32-41. 